\title{
Precurved catheters for transbrachial coronary arteriography ${ }^{1}$
}

\author{
George G. Rowe \\ From the Cardiovascular Research Laboratory, University of Wisconsin Medical School, \\ 1300 University Avenue, Madison, Wisconsin 53706, U.S.A.
}

\begin{abstract}
$A$ series of precurved catheters has been designed for use in coronary arteriography through the brachial artery. They facilitate entry of the coronary arteries, especially the left, from abnormal aortas. Their use has reduced the necessity for flushing the sinus of Valsalva as a method for viewing the coronary arteries, improved the quality of films obtained in difficult cases, and reduced fluoroscopic manipulation.
\end{abstract}

Coronary arteriography has become increasingly popular in recent years, and during this time there have developed two approaches to cannulation of the coronary arteries: that developed by Sones and his associates using the brachial artery (Sones and Shirey, 1962) and a flexible tipped catheter which is manipulated into the coronary arteries; and the other approach using the femoral artery and specially shaped catheters (Ricketts and Abrams, 1962; Judkins, 1967). The precurved catheters are shaped so that the catheter tip springs into the coronary artery when it encounters the orifice of the vessel and remains in place until it is removed (Judkins, 1967).

Those who choose the brachial artery approach to coronary arteriography can usually enter both coronary arteries without difficulty, but find on some occasions that one coronary artery, especially the left, is difficult to enter. Indeed, at times they may have to accept flushing of the sinus of Valsalva rather than entry of the vessel itself. This may compromise the quality of the pictures obtained and impair diagnostic accuracy. To circumvent this problem precurved catheters have been designed for use through the brachial artery. It was intended to incorporate the facility of entry of the coronary artery which is supplied by the precurved catheter technique (Judkins, 1967), and to ensure that once the coronary artery has been entered the catheter tip will remain engaged in it until deliberately extracted.

Received 9 January 1970.

1 This study was supported in part by grants from the U.S.P.H.S., National Heart Institute Grants.

\section{Methods}

A series of wires was bent and tried until the configurations which are shown in Fig. I were settled upon. These wires are made of stainless steel $^{2}$ and have sufficient body that once they are formed they tend to retain their shape. They are inserted into the tip of a catheter made of a material which softens in boiling water and when cooled tends to harden and retain the shape in which it has been held. This technique of moulding was copied after Judkins, and the catheters used are reshaped 'Judkins' coronary arteriography catheters' ${ }^{3}$ (Judkins, 1967). The mandrils nos. I, 2, and 3 shown in Fig. I are designed to shape a catheter so it will enter the left coronary artery, as shown in Fig. 2, from an aorta of progressively increasing diameter. The two further wires nos. 4 and 5 have also been used for entering the left (Fig. 3) and occasionally the right coronary artery in subjects in whom catheterization is difficult.

The technique of inserting the precurved catheters through the brachial and into the coronary artery is similar to that with any other catheter except that there is an increased tendency for them to become caught in side branches. When this happens they are withdrawn slightly, rotated, and advanced again until smooth entry of the aortic arch is achieved. Since the catheters from which they are made were designed for percutaneous introduction, a Teflon-coated wire guide of the standard variety (Judkins, 1967) or a 'J-guide' (Judkins et al., 1967) may be used to facilitate their introduction. Once the catheter has entered the aortic arch, it is usually easily manipulated into the ascending aorta. With the

${ }^{2}$ Wilstabrite Stainless Wire (chromium-nickel alloy No. 17, diam. 0.039 inch hard temper). Malin Company, Cleveland, Ohio, U.S.A.

${ }^{3}$ Ducor Disposable Guided Catheter. Cordis Corporation, Miami, Florida, U.S.A. 
patient in $45^{\circ}$ of left anterior oblique, the tip of the catheter is turned toward the left coronary artery. On some occasions the catheter enters the left coronary artery at once when turned towards it. On other occasions, enough of the catheter's curve has been lost during insertion into the ascending aorta that those of shapes 1,2 , and 3 need to be pressed into the sinus of Valsalva to raise the tip cephalad to the orifice of the left coronary artery. If the tip of the catheter rises above the orifice of the coronary artery it is gently manoeuvred in the aorta so that the drag of the tip on the vessel wall straightens out the catheter and depresses its tip closer to the sinus of Valsalva. When the tip of the catheter encounters the orifice of the coronary artery it tends to spring immediately into the vessel. The catheter should then rest in the coronary artery, as shown in Fig. 2. Provided that there is no decrease in the pressure measured at its tip, the catheter may remain in the left coronary artery until filming is done. On some occasions the pressure curve becomes damped while the catheter tip is against the aortic or coronary arterial wall. This may be avoided, if desired, by adding side-holes near the catheter tip. Once in the coronary artery the catheter position is watched fluoroscopically when the patient is rotated from one oblique to the other. There is probably more tendency for the catheter tip to come out of the coronary artery with this technique than there is with the Judkins percutaneous femoral technique because of the relatively greater mobility of the shoulder girdle on the trunk. However, there is a strong tendency for the catheter to remain in place with only minor amounts of adjustment. Furthermore, if the catheter does become disengaged usually it is easily manipulated into the vessel again.

If the cross-section of the aorta is too small and the sinus of Valsalva not sufficiently deep, a precurved catheter may not rest properly across the aorta with its tip in the left coronary artery. However, in this circumstance there should be no problem with catheterizing the coronary artery using the Sones technique, and as long as the present catheters are used only when the Sones technique is difficult, this problem should not arise. On the other hand, if the cross-section of the aorta is too great, even though an adequate curve is obtained, the left coronary artery may still be too far away for the small precurved catheter to reach. Under these circumstances a larger precurved catheter (no. 2 or 3 ) than those seen in Fig. $I$ is used or curves 4 and 5 may be substituted.

Precurved catheters in the shape produced by mandrils nos. 4 and 5 tend to curve in the ascending aorta with the tip of the catheter against one side and the large bend of the catheter against the other. They are then rotated so that their tip swings across the orifice of the coronary artery which has been selected for cannulation. When the tip is engaged in the coronary artery the catheter is advanced very slightly to ensure a stable position within the vessel. As with any catheter whose natural curve determines how tightly it enters the vessel, pressure from the tip must be carefully

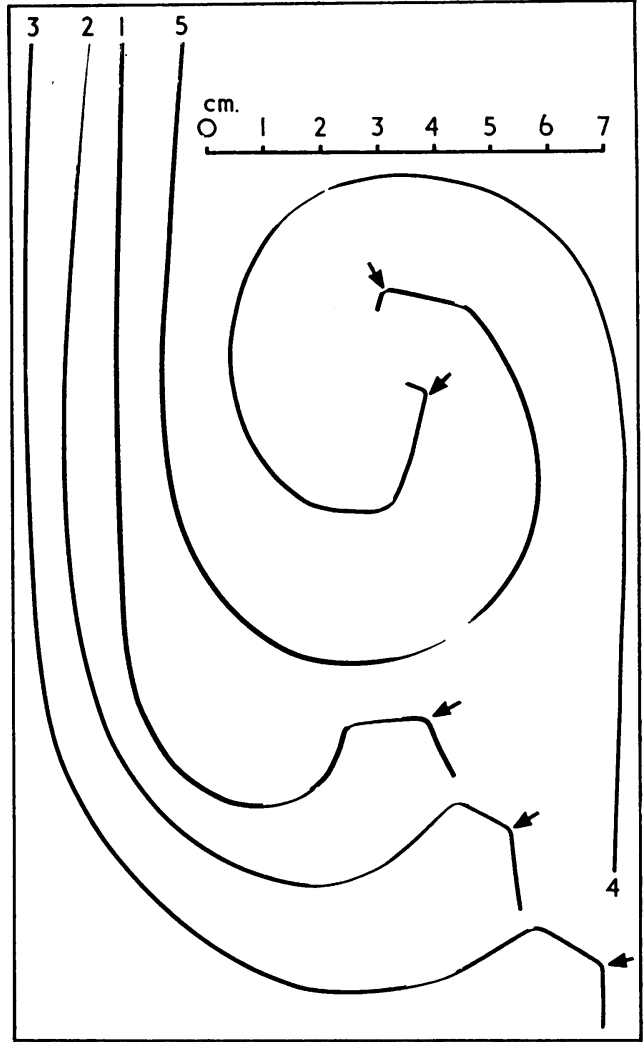

FIG. I The curved wires illustrated serve as mandrils to shape catheters so they will 'seek' the orifice of the coronary arteries, especially the left. They are designed for use in average size (no. I) and enlarged or tortuous aortic roots. The catheter tip fits snugly against the final sharp curve indicated by the arrow. The catheters are heated with the mandril in place, as described in the text, and when cooled retain the shape they have been given.

monitored to prevent inadvertent wedging in the coronary artery with consequent obstruction of blood flow. Data were collected in I I consecutive patients in whom both the Sones and precurved catheters were used. In these subjects precurved catheters were used for one or more of the following reasons: ( $I$ ) entry of the left coronary artery was difficult, (2) entry was not deep enough to get good filling of the coronary artery, (3) the catheter came out of the artery repeatedly during injection providing inadequate filling. The Table reveals the fluoroscopy time required for entry of the left coronary artery with each catheter.

\section{Results and discussion}

These catheters have now been in use for about two years. They have been used only in those subjects in which entry of a coronary 
TABLE Comparison of fluoroscopy times using Sones and precurved catheters*

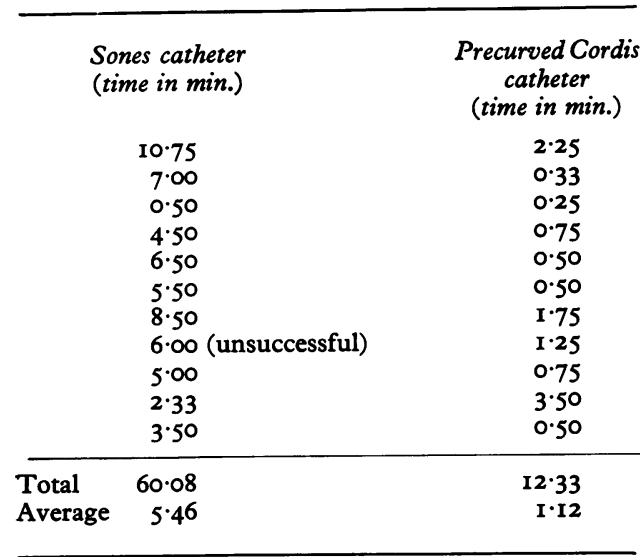

* Fluoroscopy time, in minutes, is given for entry of the left coronary artery of the same subject during the same catheterization procedure using the Sones and the precurved catheter. In no case was the filming considered optimal with the Sones catheter (see text) or the precurved catheter would not have been used.

artery has proved difficult or when the catheter comes out of the coronary artery each time contrast material is injected. Though the catheters have not invariably been used successfully, they have increased the frequency with which the coronary artery is entered in these difficult subjects, and have tended to remain in the coronary artery with relatively little tendency to come out during turning the patient from one oblique projection to the other. Since the introduction of these catheters, coronary arteriography has been accomplished more expeditiously. The quality of the pictures obtained has been improved, and the necessity for flushing the sinus of Valsalva as a method to opacify coronary arteries has been reduced. It is apparent from the Table that entry of the left coronary artery was facilitated in these subjects by the use of the precurved catheter. It should be emphasized, however, that these are selected subjects in whom use of the precurved catheter was considered to be necessary.

The precurved catheter enters the left coronary artery so easily on most occasions that its use would be the procedure of choice if it were not for the greater versatility of the Sones catheter. Thus the Sones catheter usually enables the left ventricle to be entered, as well as both coronary arteries, without difficulty. Since the present, precurved, catheters tend to assume their natural shape in the ventricle, they tend to produce premature contractions as soon as they enter the left ventricle and may have to be withdrawn

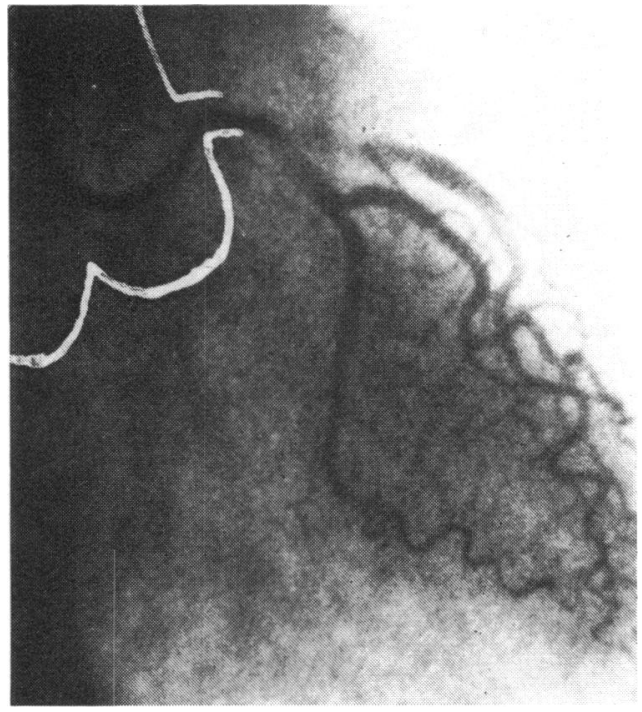

FIG. $2 A$ precurved catheter (no. I, Fig. I) is shown in the left coronary artery of a subject in whom satisfactory pictures had not been obtained because the catheter did not stay well in the coronary artery. Once introduced it remained throughout filming in both right and left oblique projections. The aorta and the sinus of Valsalva have been sketched in in white ink to display the relation of the catheter to these structures.

FIG. 3 A precurved catheter (no. 5, Fig. I) is shown in position in the left coronary artery. The semi-vertical ascent of the left coronary artery from the aortic root made it difficult for us to cannulate the orifice of this artery with usual catheterization technique. The precurved catheter remained in this vessel during rotation from the left to the right oblique.

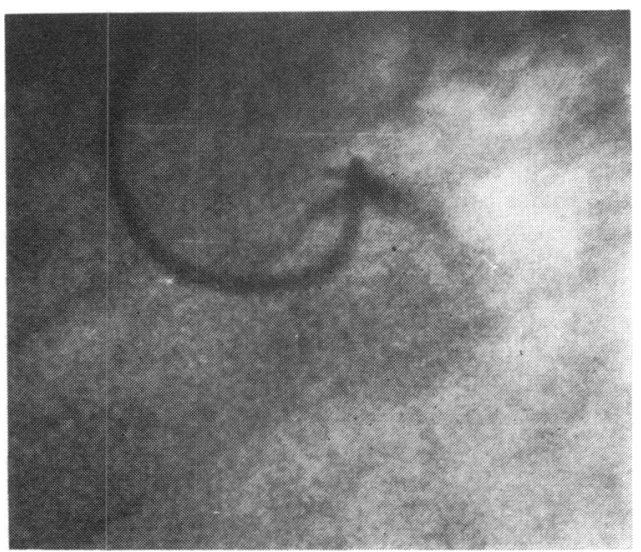


into the aorta. Consequently we try to do the entire procedure with the Sones catheter, except for the left ventricular opacification, where N.I.H. catheters are used. If coronary arteriography is accomplished smoothly, precurved catheters are not used. If we are unable to enter a coronary artery, or to obtain adequate pictures because of a tendency for the catheter to come out of the artery during injection of contrast material, a precurved catheter no. 1, 2, or 3 (Fig. I) is used. Though not invariably successful, they are likely to be. If they are not helpful a no. 4 or 5 catheter is used.

It must be emphasized that these preformed catheters are of greatest use in subjects with an abnormal aortic arch because of dilatation and/or tortuosity. It is in precisely these subjects where the greatest danger of arterial injury exists because of their extensive atherosclerosis and because the tip of the catheter tends to press against the side of the artery rather than to lie free in its lumen. As already indicated, flexible guide wires may reduce this hazard. Though dissection of the aorta or the coronary arteries has not been produced with these catheters to date, it is regarded as a calculated risk. Dissection of the coronary arteries is a perennial risk with coronary arteriography, but has been reported to be more likely to occur with the use of the transfemoral precurved catheters (Haas, Peterson, and Jones, 1968). Unless the present catheters are used with care the same complication may occur.

\section{References}

Haas, J. M., Peterson, C. R., and Jones, R. C. (1968). Subintimal dissection of the coronary arteries. A complication of selective coronary arteriography and the transfemoral percutaneous approach. Circulation, 38, 678.

Judkins, M. P. (1967). Selective coronary arteriography. Part I: A percutaneous transfemoral technic. Radiology, 89, 815.

—, Kidd, H. J., Frische, L. H., and Dotter, C. T. (1967). Lumen-following safety J-guide for catheterization of tortuous vessels. Radiology, 88, II27.

Ricketts, J. H., and Abrams, H. L. (1962). Percutaneous selective coronary cine arteriography. fournal of the American Medical Association, 181, 620.

Sones, F. M., Jr., and Shirey, E. K. (I962). Cine coronary arteriography. Modern Concepts of Cardiovascular Disease, 31, 735. 